\title{
THE EXTREMAL FUNCTIONS FOR CERTAIN PROBLEMS CONCERNING SCHLICHT FUNCTIONS
}

\author{
BY ARTHUR E. OBROCK ${ }^{1}$ \\ Communicated by Maurice Heins, February 12, 1965
}

1. Let $S$ denote the classical family of schlicht functions on the unit disk normalized by the conditions $f(0)=0, f^{\prime}(0)=1$. Under a suitable metric such as $d(f, g)=\sup \{f(z)-g(z)|:| z \mid=1 / 2\}$ it is a compact metric space. Let $0<r<1$. We are interested in the closed subspaces $B_{r}=\{f \in S:|f(z)|<1 / r\}$ and $C_{r}=\{f \in S: f(z) \notin D(f)\}$, where $D(f)$ is a domain of outer conformal radius $1 / r$ with respect to the point at infinity. The general problem is to determine the explicit region of values $V(T)$ of certain continuous functions $T$ from one of these spaces $F$ into some manifold $M$. We also ask what (extremal) functions in $F$ are mapped by $T$ into $\partial V(T)$, the boundary of $V(T)$. In particular consider the function

$\left(^{*}\right) \quad T(f)=\left(f^{0}\left(z_{1}\right), f^{1}\left(z_{1}\right), \cdots, f^{n_{1}}\left(z_{1}\right), \cdots, f^{0}\left(z_{m}\right), \cdots, f^{n_{m}}\left(z_{m}\right)\right)$,

where $f^{k}\left(z_{j}\right)=H\left(f, z_{j}, k\right)$ denotes the value of the $k$ th derivative of $f$ at $z_{j}$, except that, for technical reasons $H$ is interpreted as a continuous function into the logarithmic covering surface when $z_{j} \neq 0$ and $k=0$ or 1 .

The well-known results for the case $F=S, m=1, z_{1}=0$, due to Spencer and Schaeffer, can be found in [10]. Royden [11] indicated the more general result when $F=S$. Their key tools were Teichmüller's Theorem [10, p. 93] and their variational method. By using Jenkins' General Coefficient Theorem [7] and a form of the Brouwer Fixed Point Theorem we are able to generalize some of their results to a somewhat wider class of functions $T$ and spaces $F$.

2. For the functions $T$ defined by $\left(^{*}\right)$ there are certain quadratic differentials $P(w) d w^{2}$, indicated by the Teichmüller Principle [8, p. 48], which we call admissible with respect to $T$. We call the pair $\left(P(w) d w^{2}, f(z)\right)$ an admissible association with respect to $T$ if $P(w) d w w^{2}$ is admissible with respect to $T, f \in F, f(\{|z|<1\})$ is an admissible domain with respect to $P(w) d w^{2}$ in the sense of Jenkins [8, p. 49], and $\left\{f\left(z_{j}\right): 1 \leqq j \leqq m\right\}$ contains the poles of $P(w) d w^{2}$ in $f(\{|z|<1\})$.

${ }^{1}$ This work was supported by an NSF Regular Postdoctoral Fellowship at the University of Illinois. It is an extension of part of the author's Ph.D. dissertation carried out under the guidance of Professor James A. Jenkins at Washington University. 
Now we can state our main theorem.

THEOREM. Let $T$ be defined by $\left(^{*}\right)$. To every boundary point $p_{*}$ of $V(T)$ there is precisely one function $f_{*}$ in $F$ such that $T\left(f_{*}\right)=p_{*}$. There is at least one admissible association with respect to $T\left(P(w) d w w^{2}, f_{*}(z)\right)$. Conversely, if $\left(P(w) d w^{2}, f(z)\right)$ is an admissible association with respect to $T$ then $T(f)$ lies in the boundary of $V(T)$.

Outline of The Proof. It is easy to show that $V(T)$ is homeomorphic to a unit ball in a Euclidean space of an appropriate dimension and that an infinite number of functions in $F$ are mapped by $T$ into every interior point of $V(T)$ by a proof analogous to the proof in $[10$, p. 9] for the (Bieberbach) coefficient region. Consequently, the last statement of our theorem follows from the equality statement of the General Coefficient Theorem. Next we introduce a topology on $W$, the set of admissible associations with respect to $T$. Then we construct a map $H_{1}: \partial V(T) \rightarrow W$ such that the composed map $H_{2} \circ H_{1}$ : $\partial V(T) \rightarrow \partial V(T)$, (where $H_{2}:\left(P(w) d w^{2}, f(z)\right) \rightarrow T(f)$ ) is homotopic to the identity relative to $\partial V(T)$. The remainder of the theorem then follows from a form of the Brouwer Fixed Point Theorem.

3. The computation of the explicit values of $T$ is in general a difficult task. One approach is to find the relations between the coefficients of $P(w) d w^{2}$ and the coefficients of $Q(z) d z^{2}=P(f(z))\left(f^{\prime}(z)\right)^{2} d z^{2}$, the induced quadratic differential. Then the values of $T(f)$ can be computed in terms of these coefficients. In simple cases we are able to obtain the explicit results in this way. For example we obtain a generalization of Grunsky's region $V(T)[5]$ (where $T(f)=\log f\left(\rho e^{i \theta}\right)$, $F=S)$ to the spaces $B_{r}, C_{r}$.

In the case $B_{r} V(T)$ is the convex region bounded by the Jordan curve $C(t)=\log (s(t) / r)+i(\theta+\phi(t))$, for $-\pi \leqq t \leqq \pi$, where $s=s(t)$ $=s(-t), \phi=\phi(t)=-\phi(-t)$, and are defined on $0 \leqq t \leqq \pi$ from the homeomorphisms indicated by the relations

$$
\cos t=\log \frac{1-\rho^{2}}{1-s^{2}} \frac{s}{r \rho} / \log \frac{1-\rho}{1+\rho} \frac{1+s}{1-s}, \phi=\sin t \log \frac{1+\rho}{1-\rho} \frac{1-s}{1+s} .
$$

The unique extremal function $f_{t} \in B_{r}$ associated with $C(t)$ is the unique function such that $f_{t}(\{|z|<1\})$ is admissible with respect to

$$
P(w) d w^{2}=-e^{-i t} \frac{\left(w-e^{i(\phi+t)} / r\right)^{2} d w^{2}}{w^{2}\left(w-s e^{i \phi} / r\right)\left(w-e^{i \phi} / s r\right)} .
$$

When such explicit solutions are determined, we can often follow Jenkins' example [9] to solve problems where $T_{1}=H \circ T$. For exam- 
ple, if $T_{1}(f)=\arg \left(f\left(\rho e^{i \theta}\right) / \rho e^{i \theta}\right)$ on $B_{r}$, then $V\left(T_{1}\right)$ is found by determining $\sup \{\phi(t) \mid 0 \leqq t \leqq \pi\}$. We find that if $f \in B_{r}$, then

$$
\left|\arg \left(f\left(\rho e^{i \theta}\right) / \rho e^{i \theta}\right)\right| \leqq\left(\log \frac{1-s_{*}}{1+s_{*}} \frac{1+\rho}{1-\rho}\right)\left(\frac{1-s_{*}^{2}}{1+s_{*}^{2}}\right),
$$

where $s_{*}=s\left(t_{*}\right)$ is the unique value of $s$ where

$$
2 \log \frac{1-\rho}{1+\rho} \frac{1+s}{1-s}=\left(s+\frac{1}{s}\right) \log \frac{1-\rho^{2}}{1-s^{2}} \frac{s}{r \rho} .
$$

Equality occurs only for the functions $f_{t^{*}}, f_{-t^{*}}$.

4. Duren and Schiffer [2], Gaier and Huckemann [3], Huckemann [6], Gehring and Hällström [4], and Duren [5] have investigated schlicht functions on an annulus. More precisely, let $F_{r}$ denote the family of schlicht functions on $r<|z|<1 / r$ such that $f(1 / \bar{z})=1 / \bar{f}(z)$, $f(1)=1$. We obtain results similar to those of the theorem in $\$ 2$ for functions $T$ on $F_{r}$ of the form $\left({ }^{*}\right)$. In the quadratic differentials $P(w) d w^{2}, P(w)$ is again a rational function of $w$. However, the computations of $T(f)$ are more complicated here due to the fact that in the induced $Q(z) d z^{2}, Q(z)$ is no longer rational in $z$ but is given by $v(\log z) / z^{2}$ where $v(w)$ is a doubly periodic function.

\section{BIBLIOGRAPHY}

1. P. L. Duren, Distortion in certain conformal mappings of an annulus, Michigan Math. J. 10 (1963), 431-441.

2. P. L. Duren and M. Schiffer, $A$ variational method for functions schlicht in an annulus, Arch. Rational Mech. Anal. 9 (1962), 260-272.

3. D. Gaier and F. Huckemann, Extremal problems for functions schlicht in an annulus, Arch. Rational Mech. Anal. 9 (1962), 415-421.

4. F. W. Gehring and G. af Hällström, A distortion theorem for functions schlicht in an annulus, Ann. Acad. Sci. Fenn. Ser. A I (1963), no. 325, 15 pp.

5. H. Grunsky, Neue Abschätzungen zur konformen Abbildung ein- und mehrfach zusammenhängender Bereiche, Schr. Deutsche Math.-Ver. 43 (1934), 140-143.

6. F. Huckemann, Über einige Extremalprobleme bei konformer Abbildung eines Kreisringes, Math. Z. 80 (1962), 200-208.

7. J. A. Jenkins, A general coefficient theorem, Trans. Amer. Math. Soc. 77 (1954), 262-280. 1958.

8. - Univalent functions and conformal mapping, Springer-Verlag, Berlin,

9. - On weighted distortion in conformal mapping, Illinois J. Math. 4 (1960), 28-37.

10. A. C. Schaeffer and D. C. Spencer, Coefficient regions for schlicht functions, Amer. Math. Soc. Colloq. Publ. Vol. 35, Amer. Math. Soc., Providence, R. I., 1950.

11. H. L. Royden, The interpolation problem for schlicht functions, Ann. of Math. 60 (1954), 326-344.

UNIVERSITY OF ILLINOIS 